\title{
SCREENING DEL MALESTAR PSICOLÓGICO EN PACIENTES CON CÁNCER DE PULMÓN NO MICROCÍTICO CON TRATAMIENTO QUIRÚRGICO
}

\author{
SCREENING OF EMOTIONAL DISTRESS IN NON-SMALL CELL LUNG CANCER \\ PATIENTS WITH SURGERY TREATMENT
}

\begin{abstract}
Patricia Pradera', Juan Antonio Cruzado², $\mathrm{M}^{a}$ Eugenia Olivares ${ }^{3}$, Florentino HernandoTrancho'
\end{abstract}

' Servicio de Cirugía Torácica. Hospital Clínico San Carlos. Madrid.

2 Facultad de Psicología. Universidad Complutense. Madrid.

${ }^{3}$ Servicio de Ginecología y Obstetricia. Hospital Clínico San Carlos. Madrid.

Resumen

El cáncer de pulmón es el tipo de enfermedad neoplásica más común y la causa más frecuente de muerte por causa oncológica en el mundo occidental. Su mal pronóstico debido a la dificultad de realizar un diagnóstico precoz, las atribuciones causales personales relacionadas con el consumo de tabaco y los síntomas derivados, no solo del progreso de la enfermedad sino también de los efectos secundarios del tratamiento, pueden provocar elevados síntomas de malestar emocional y una disminución de la calidad de vida.

El propósito de este artículo es revisar en primer lugar la problemática psicológica encontrada en los pacientes afectados de cáncer de pulmón no microcítico susceptibles de intervención quirúrgica, y en segundo lugar, proporcionar información acerca de los procedimientos de evaluación más frecuentemente utilizados en este grupo de pacientes con el objetivo de poder establecer un protocolo de screening que permita detectar aquellos pacientes en riesgo de presentar malestar emocional.

En base a los datos existentes se recomienda la evaluación de las variables malestar emo-

\section{Abstract}

Lung cancer is the most common malignant disease and the principal cause of oncology death in Western countries. Its poor prognosis due to the difficulty of early diagnosis, causal attributions related to personal tobacco consumption and resulting symptoms, not only from the progress of the disease but also from the side effects of treatment, can cause high emotional distress and a decreased quality of life.

The purpose of this paper is to review first the psychological problems found in patients suffering from non-small lung cancer susceptible to surgery treatment, and secondly, provide information about the assessment procedures most frequently used in this group of patients in order to establish a screening protocol to detect patients at risk for emotional distress.

Based on existing data we recommended to assess emotional distress, pain and quality of life from the time of diagnosis and through all the process with the following assessment tools: Hospital Anxiety and Depression Scale, EORTC QLQ C30 and LC13, and Brief Pain Inventory. With the

\section{Correspondencia:}

Patricia Pradera

Unidad de Cirugía Torácica.

Hospital Clínico San Carlos. C/ Profesor Martín Lagos

28040 Madrid

Email: p.pradera@institutooncover.com 
cional, calidad de vida y dolor desde el momento del diagnóstico y a lo largo del proceso con los instrumentos de evaluación: Escala de Ansiedad y Depresión Hospitalaria, EORTC QLQ-C30 y LC13, y Cuestionario Breve del Dolor. Con los resultados del screening se debería derivar aquellos pacientes que presenten malestar a la unidad de Psicooncología.

Palabras clave: Screening, cáncer de pulmón, malestar emocional, calidad de vida, dolor, evaluación psicológica, afrontamiento, apoyo social y familiar. results of the screening measure we should refer patients experiencing to the PsychoOoncology Unit.

Key Words: Screening, lung cancer, psychological distress, quality of life, pain, psychological assessment, coping, social and family support.

\section{INTRODUCCIÓN}

El cáncer de pulmón afecta a 82 de cada 100.000 españoles. En España se diagnostican cada año 20.000 nuevos casos. La enfermedad representa el $12 \%$ de todos los tipos de tumores y el $20 \%$ de las muertes asociadas a esta patología en nuestro país ${ }^{(1)}$.

Histológicamente se divide en dos grandes grupos: microcítico o de células pequeñas (SCLC) que representa aproximadamente el 25\% y, el cáncer de células no pequeñas o no microcítico (NSCLC) que se diagnostica en el $75 \%$ de los casos. Cada tipo histológico difiere en sus características clínicas, respuesta al tratamiento y pronóstico ${ }^{(2)}$. El presente artículo se centra exclusivamente en el cáncer de pulmón no microcítico susceptible de tratamiento quirúrgico con el objetivo de proporcionar información específica de este grupo de pacientes.

Existen varios factores de riesgo relacionados con el desarrollo del cáncer de pulmón independientemente de su tipo histológico. El consumo activo de tabaco es la causa fundamental del desarrollo de la enfermedad. En este sentido, se ha observado que los hombres poseen una tasa de cáncer de pulmón tres veces mayor que las mujeres. Esto es debido a que la frecuencia en la conducta de fumar es mayor en $\operatorname{estos}^{(3)}$.
En la corriente principal del humo del tabaco se han identificado al menos 4.700 compuesto químicos, de los cuales 78 han sido considerados carcinógenos probados por la IARC (International Agency for Research on Cancer) $)^{(4)}$. La nicotina, principal responsable de la adicción al tabaquismo, podría tener un cierto efecto carcinógeno. Los carcinógenos del tabaco, no solo actúan como inductores de alteraciones genéticas sino también en el aumento de la progresión del tumor ${ }^{(5)}$. Un consumidor habitual tiene 20 veces más riesgo de desarrollar cáncer de pulmón que un no fumador $^{(6)}$.

Dejar de fumar después del diagnóstico está asociado a mejoras en el tiempo de supervivencia, en la calidad de vida, en la respuesta al tratamiento, a un descenso de complicaciones post-quirúrgicas, aparición de otros tumores primarios y metástasis, y al descenso de complicaciones tras el tratamiento ${ }^{(7)}$.

Por otro lado, existen factores genéticos que explicarían una mayor susceptibilidad individual a desarrollar cáncer de pulmón. Las alteraciones genéticas están implicadas en la susceptibilidad a los carcinógenos pulmonares y en la carcinogénesis. Se han descrito mutaciones en los genes K-ras y p53 en el $50 \%$ de los pacientes que padecen este tipo de cáncer ${ }^{(8)}$.

Asimismo, algunas investigaciones han 
señalado que padecer cardiopatías, diabetes mellitus u obstrucción pulmonar crónica representa un factor de riesgo. Por ejemplo, la enfermedad pulmonar obstructiva crónica se asocia con dificultades en la aclaración de carcinógenos debido a la inflamación crónica de la mucosa ${ }^{(9)}$. Es importante destacar que la comorbilidad es especialmente alta en los pacientes con cáncer de pulmón comparado con otros pacientes con cáncer ${ }^{(10)}$.

Por último, se conocen numerosos agentes laborales y ambientales capaces de favorecer el desarrollo del cáncer de pulmón como el gas radón, asbesto, uranio, cadmio, cromo, níquel, arsénico y terpenos ${ }^{(11)}$.

Una de las razones por las cuales el cáncer de pulmón está asociado a una alta mortalidad es su difícil diagnóstico precoz. El diagnóstico de sospecha está presente en todo paciente fumador de más de 40-45 años que consulta por clínica respiratoria o sistémica de causa no aclarada ${ }^{(12)}$.

Los síntomas más frecuentes encontrados son la tos, la fatiga, el dolor, la disnea, la disminución del apetito, el insomnio, la pérdida de peso y la fiebre ${ }^{(13)}$. También es frecuente la aparición de una infección respiratoria o una neumonía que no cede tras haber cumplimentado adecuadamente un tratamiento antibiótico ${ }^{(14)}$.

Las pruebas que se realizan habitualmente para el diagnóstico son análisis de sangre y orina, radiografía de tórax, citología de esputo, mediastinoscopia, broncoscopia o fibrobroncoscopia, punción aspirativa con aguja fina, tomografía axial computarizada (TAC) y tomografía por emisión de positrones (PET) ${ }^{(15)}$. En la actualidad, no existen pruebas que cumplan los requisitos necesarios para ser utilizadas como screening del cáncer de pulmón en la población general.

El siguiente paso tras el diagnóstico es la estadificación, cuyo objetivo es estimar el pronóstico y decidir el tipo de trata- miento en base al sistema de estadificación internacional TNM ${ }^{(16)}$.

Una vez realizado el diagnóstico de NSCLC y realizada la estadificación del mismo, se establece el tratamiento en base a las características del paciente, a su enfermedad y a los protocolos existentes. El tratamiento quirúrgico sigue siendo el tratamiento de elección y el de mayor expectativa de curación para NCSL ${ }^{(17)}$. Sin embargo, a día de hoy, se estima que solo entre el 20 y el $30 \%$ de los pacientes son susceptibles de tratamiento quirúrgico con criterio curativo en el momento del diagnóstico ${ }^{(18)}$.

La cirugía tiene como objetivo fundamental la extirpación integral de la enfermedad, por lo que el tipo de resección está en función del tamaño y localización del tumor, así como de su extensión a estructuras vecinas (pared torácica, diafragma y pericardio). Las intervenciones quirúrgicas más habituales son resección segmentaria, lobectomía, bilobectomía y neumonectomía ${ }^{(19)}$.

Los factores que determinan la operabilidad son la edad, el estado clínico, la función pulmonar, la función cardiovascular y la comorbilidad asociada grave ${ }^{(20)}$. La edad media de los pacientes sometidos a tratamiento quirúrgico supera los 62 años y se ha incrementado en la última década. Actualmente, en pacientes mayores de 80 años sólo los estadios I y II son factibles de intervención quirúrgica siempre que no precisen neumonectomía ${ }^{(21)}$.

En el estudio realizado por Pagano et al. ${ }^{(22)}$ con 2.298 pacientes con cáncer de pulmón divididos en estadio y tipo de tratamiento encontraron que en los estadios iniciales la presencia de comorbilidad, una edad avanzada y un nivel bajo educacional se asocian con una menor probabilidad de acceder a tratamiento quirúrgico.

La cirugía del cáncer de pulmón no está exenta de complicaciones y de efectos adversos. Habitualmente, no suponen 
un problema grave para el paciente, pero si alteran su calidad de vida. Las posibles complicaciones dependen de la extensión de la cirugía y la condición de salud de la persona. Los efectos secundarios más comunes la cirugía de cáncer de pulmón son el dolor, la debilidad en el tórax, en el brazo y la pérdida de la función pulmonar ${ }^{(23)}$.

En la figura 1 se presenta de manera esquemática los pasos necesarios para realizar un diagnóstico en pacientes con sospecha de cáncer de pulmón.

\section{EL DOLOR}

El dolor crónico es una de las complicaciones más comunes después del tratamiento quirúrgico. El Síndrome de Dolor Post-torácico se define como un dolor que persiste o recurre a lo largo de la incisión de la toracotomía por como mínimo dos meses después de la intervención quirúrgica y cuya causa más común es un traumatismo en el nervio intercostal durante la toracotomía(24).

Se da en más del $80 \%$ de los pacientes, de los cuales un 5\% puede padecer un dolor severo ${ }^{(25)}$. Wildgaard et al. ${ }^{(26)}$ Ilevaron a cabo una investigación cuyo objetivo era obtener información sobre la prevalencia del síndrome de dolor post-torácico tras la cirugía de una muestra de 546 pacientes a los cuales se les siguió durante 22 meses. Entre el 4 y el $12 \%$ presentaron un dolor severo, correspondiéndose los datos con lo encontrado en la bibliografía.

La mayoría de los pacientes experimentan dolor a lo largo de toda la cicatriz, pero el dolor también puede ocurrir en otros lugares como en el pecho, en la espalda o en más de una localización. Algunos factores que se han identificado como

Figura 1.

\section{Organigrama del proceso de diagnóstico y tratamiento de cáncer de pulmón.}

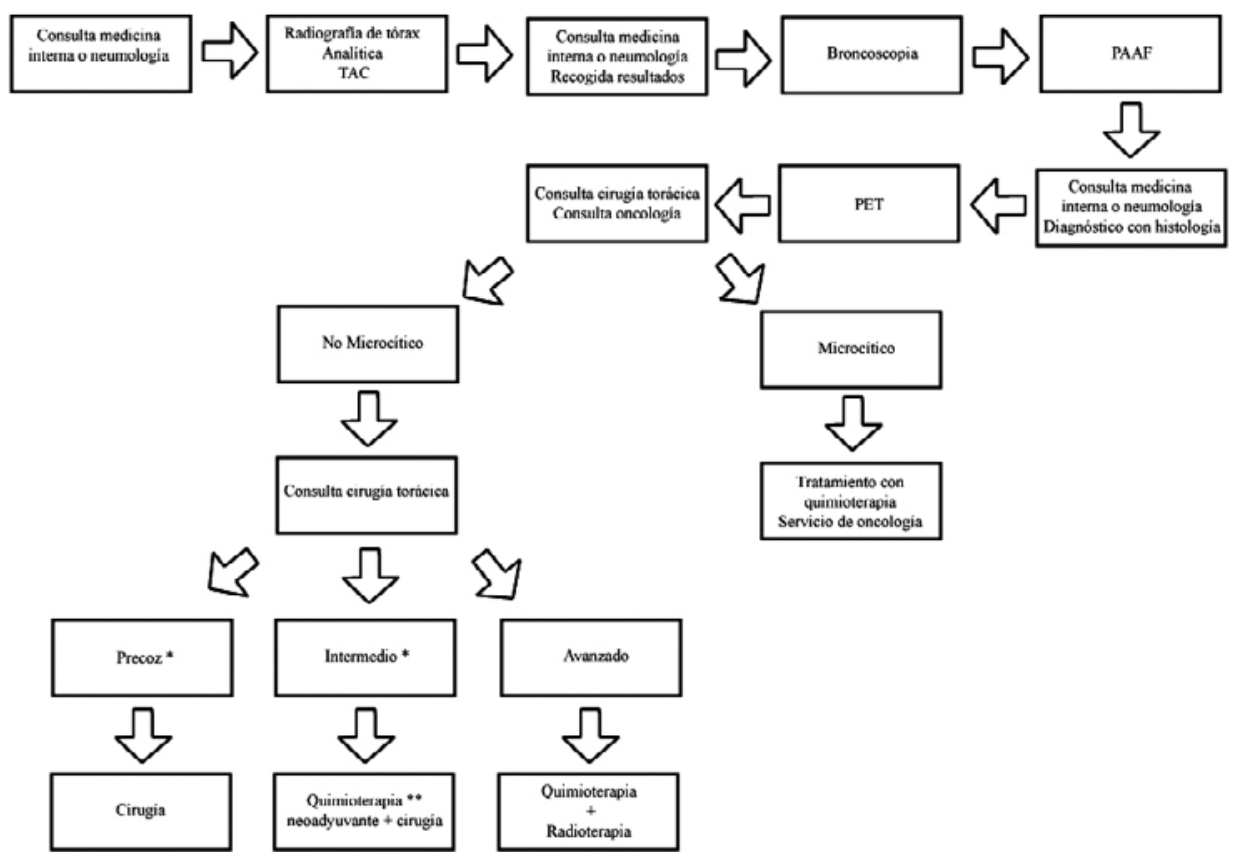

- Si hay afectación ganglionar se debe realizar primero un tratamiento con quimioterapia.

*- Entre quimioterapia y cirugia se realica una reestadificación a través de un PET o mediastinoscopia. 
predictores son la intensidad del dolor después de las primeras 24 horas, el consumo de analgésicos durante el primer día postoperatorio y durante la primera semana ${ }^{(27)}$. A su vez, también se han descrito factores que agravan o inducen el dolor como el estrés emocional, la ansiedad, el cambio del tiempo, actividades como coger objetos pesados, acostarse sobre el lado operado, estar mucho tiempo sentado y trabajar con la mano del lado afectado(28).

El dolor es subjetivo y está influenciado por aspectos culturales y variables psicológicas. Por este motivo, es esencial llevar a cabo una evaluación y preparación psicológica completa ${ }^{(29)}$. Las escalas analógicas visuales resultan de gran utilidad en la evaluación del dolor. Igualmente, un instrumento frecuentemente utilizado en este grupo de pacientes es el Cuestionario Breve del Dolor (Brief Pain Inventory, BPI) desarrollado por Cleeland en $1991^{(30)}$ y adaptado a la población española por Badía et al. en $2003^{(31)}$. Está constituido por 15 preguntas que hacen referencia a dos dimensiones básicas, la intensidad del dolor y la inferencia del dolor en las actividades diarias del paciente. La primera, formada por cuatro ítems, permite determinar niveles de intensidad del dolor y la segunda, formada por siete preguntas, permite conocer los efectos del dolor sobre la actividad general, estado de ánimo, capacidad para caminar, trabajo habitual, relaciones con otras personas, sueño y disfrute de la vida. Además de las dos dimensiones reseñadas, el cuestionario consta de otras preguntas que evalúan el nivel de alivio que el tratamiento para el dolor proporciona al paciente y la localización de este. Una mayor puntuación en las preguntas indica una mayor intensidad e interferencia del dolor. Su aplicación es breve, aproximadamente unos $15 \mathrm{mi}$ nutos.

Es de gran importancia que la evaluación del dolor se repita en varias ocasiones durante el tratamiento puesto que puede sufrir modificaciones a lo largo de todo el proceso. Una completa explicación de la cirugía y de las expectativas puede resultar eficaz en la reducción de la ansiedad post-operatoria y el uso de medicamentos para el dolor.

\section{MALESTAR EMOCIONAL}

Aproximadamente el $40 \%$ de los pacientes diagnosticados con cáncer de pulmón experimentan malestar emocional ${ }^{(32)}$. La sintomatología ansioso-depresiva es la más frecuente y puede ser parte de la reacción al diagnóstico, pero en muchos pacientes esta persiste causando una carga adicional durante el tratamiento, conduciendo a mayores dificultades en el manejo general de la enfermedad y en el control de los síntomas, incrementando la estancia hospitalaria y disminuyendo la satisfacción con el tratamiento(33).

Algunos estudios han evaluado los posibles factores de riesgo de padecer malestar emocional en este grupo de pacientes. Centrados en aspectos sociodemográficos, Uchitomi et al. ${ }^{(34)}$ concluyen en su estudio que la incidencia de depresión un año después de la cirugía se asocia con el nivel educativo, además de con la presencia de sintomatología depresiva en el diagnóstico. Respecto al género, Sarna et al. ${ }^{(35)}$ determinan que las mujeres diagnosticadas de neoplasia maligna de pulmón experimentan más ansiedad y mayor preocupación después del diagnóstico que los hombres, particularmente en referencia a la gestión de las tareas domésticas y el cuidado de sí mismas. Por su parte, Graves et al. ${ }^{(36)}$ indican que una edad más joven se asocia con niveles más elevados de malestar y un mayor deseo de recibir ayuda para los síntomas derivados de la enfermedad y el tratamiento.

En referencia a la influencia de las características clínicas, Hopwood y Ste- 
phens $^{(37)}$ muestran que los pacientes con mayor sintomatología depresiva eran aquellos que mostraban un estado funcional disminuido, más síntomas físicos respiratorios, de tos, dolor y cansancio severos. Más de la mitad de los pacientes con sintomatología depresiva continuaban presentándola en el seguimiento, lo que sugiere que la morbilidad no es una simple reacción transitoria al diagnóstico. Uchitomi et al. ${ }^{(38)}$ indican que los síntomas depresivos encontrados en su estudio a los tres meses correlacionaban con el dolor y el estado funcional. De forma similar, el estudio de Smith et al. ${ }^{(39)}$ indica que niveles más elevados de disnea correlacionaban significativamente con niveles elevados de dolor y sintomatología ansiosa. Asimismo, en su estudio, Handy et al. ${ }^{(40)}$ encontraron que tras seis meses de la intervención quirúrgica de pulmón, los pacientes presentaban una disminución significativa a nivel físico, social y mental así como dolor físico. Pacientes con una peor difusión de la capacidad pulmonar en la medición de monóxido de carbono, tenían peor funcionamiento físico, estado psicológico, espiritual y calidad de vida en la fase pre y postoperatoria. Por su parte, Kurtz et al. ${ }^{(41)}$ muestran que la severidad de los síntomas físicos se relacionaban con sintomatología depresiva. Mayor severidad de los síntomas correspondía con niveles más elevados de sintomatología depresiva. De manera similar, Henoch et al. ${ }^{(42)}$ encontraron en su estudio que la sintomatología depresiva en este grupo de pacientes correlacionaba con la discapacidad física y un empeoramiento del estado funcional, lo que indica que una proporción sustancial de la depresión en estos pacientes estaba relacionada con la gravedad de la enfermedad subyacente. Por último, Buchanan et al. ${ }^{(43)}$ encontraron correlaciones positivas entre ansiedad y cierta sintomatología física como disnea, tos y hemoptisis. A mayor sintomatología ansiosa, se observó un descenso en el funcionamiento físico y peor pronóstico.

Relacionado con el consumo de tabaco, LoConte et al. ${ }^{(44)}$ concluyeron que una historia de consumo de tabaco correlacionaba con un incremento en los niveles de culpabilidad y vergüenza independientemente del tipo de tumor. Una identificación de las conductas del pasado como contribuyentes al desarrollo de la enfermedad correlacionaba con niveles de culpabilidad, vergüenza, sintomatología ansiosa y depresiva más elevados.

A la hora de realizar la evaluación del malestar emocional en este grupo de pacientes, se debe tener en cuenta que los síntomas vegetativos de la depresión son síntomas físicos experimentados frecuentemente por los pacientes con cáncer, en especial la astenia, la actividad motora ralentizada, el insomnio, la ausencia de libido, la anorexia y la pérdida de peso. Por este motivo es importante prestar especial atención a los síntomas psicológicos como humor disfórico, sentimiento de inutilidad, indefensión, culpabilidad, pérdida de la autoestima, anhedonia o desesperanza ${ }^{(45)}$.

En esta misma línea se puede incluir el síndrome de desmoralización. Este síndrome se caracteriza por: 1) síntomas afectivos de angustia existencial que incluyen desesperanza; 2) actitudes cognitivas de pesimismo, indefensión, sensación de estar atrapado, fracaso personal o ausencia de un futuro que merezca la pena; 3) ausencia conativa del impulso o motivación para actuar de un modo diferente; 4) características asociadas de alienación social o aislamiento y ausencia de apoyo; 5) fluctuaciones en la intensidad emocional durante más de dos semanas; 6) ausencia de depresión mayor o de otro trastorno psiquiátrico como condición principal(46).

El principal instrumento de evaluación del malestar emocional utilizado por las 
investigaciones descritas en este apartado es la Escala de Ansiedad y Depresión Hospitalaria (Hospital Anxiety and Depression Scale, HADS), diseñada por Zigmond y Snaith en $1983^{(47)}$ y validada al español por Tejero et al. en 1986(48). Está compuesta de catorce ítems repartidos en dos subescalas, con siete ítems cada una. Las escalas evalúan los síntomas clínicos de la ansiedad, así como los síntomas clínicos de la depresión. También, el Inventario de Ansiedad Estado-Rasgo (State-Trait Anxiety, STAI) es frecuentemente utilizado. Diseñado por Spielberger et al. en $1970^{(49)}$ y adaptado a la población española por Spielberger et al. en $1982^{(50)}$. Consiste en una escala tipo Likert con dos escalas que contienen 20 elementos cada una. Mide dos tipos de ansiedad, la ansiedad estado y la ansiedad rasgo. Su tiempo de aplicación es de aproximadamente unos 15 minutos. Por su parte, el Inventario de Ansiedad de Beck (Beck Anxiety Inventory, BAI) fue diseñado por Beck et al. en $1988^{(51)}$ y adaptado al castellano por Sanz y Navarro en $2003^{(52)}$. Está diseñado específicamente para reducir el solapamiento entre la depresión y escalas de ansiedad mediante la medición de los síntomas de ansiedad mínimamente compartidos con los de la depresión. Su tiempo de aplicación también es muy breve, aproximadamente entre 5 y 10 minutos.

En la evaluación de síntomas depresivos destacan los siguientes instrumentos de evaluación. El Cuestionario Perfil de Estados de Humor (Profile of Mood States, POMS) es frecuentemente utilizado. Creado por Mcnair et al. en $1971^{(53)}$ y adaptado a la población española por Arce et al. en $2002^{(54)}$. Con él se obtiene un índice general de alteración del estado de ánimo y siete medidas parciales: tensión, depresión, cólera, vigor, fatiga, confusión y amistad. Su tiempo de aplicación es de 10 minutos. La Escala del Centro de Estudios Epidemiológicos de la Depresión (Center for Epidemiologic Studies-Depression Scale, CES-D) fue creada en 1977 por Laurie Radloff ${ }^{(55)}$. Junto con el instrumento anterior es uno de los más frecuentemente utilizados. Se centra fundamentalmente en los componentes cognitivo conductual de la sintomatología depresiva, con menor presencia de síntomas somáticos. Consta de 20 preguntas que se encuadran dentro de nueve grupos de síntomas (anhedonia, tristeza, fatiga, agitación, ideación suicida, apetito, sueño, concentración y sentimientos de inutilidad). La traducción española ha sido realizada por Chorot y Sandín en $1991^{(56)}$.

El Inventario Breve de Síntomas desarrollado por Derogatis y Melisaratos en $1983^{(57)}$ (Brief Symptoms Inventory, BSI) y validado en nuestro país por Aragon et al. en el $2000^{(58)}$ es una medida de autoinforme de 53 ítems. El BSI es la forma abreviada del Cuestionario de los 90 síntomas (Derogatis Symptom Checklist, SCL-90$\mathrm{R})^{(59)}$. Los ítems se encuentran agrupados en 9 dimensiones: somatización, obsesióncompulsión, sensibilidad interpersonal, depresión, ansiedad, hostilidad, ansiedad fóbica, ideación paranoide y psicoticismo. Existen cuatro ítems del BSI (falta de apetito, insomnio, pensamientos sobre la muerte y sentimientos de culpa) que no se han podido asignar a ninguna dimensión específica porque tienen pesos factoriales altos en más de una. Estos ítems se incluyen en una categoría denominada «ítems adicionales» y se han mantenido en el inventario por su marcado interés clínico. El BSI contiene también tres índices globales que permiten obtener en una sola puntuación el grado general de malestar psicológico del individuo. El «Índice global de gravedad» (Global Severity Index, GSI), concebido como el mejor indicador de los tres, combina el número de síntomas y la intensidad del malestar percibido, y se obtiene dividiendo la puntuación total entre el número de ítems del instrumento. Su 
tiempo de aplicación es de aproximadamente 10 minutos.

El Inventario de Depresión de Beck (Beck Depression Inventory, BDI) fue creado por Beck et al. en $1979^{(60)}$ y revisado en $1996^{(61)}$ y adaptado al castellano por Vázquez y Sanz en $1999^{(62)}$. Es un cuestionario autoadministrado que consta de 21 preguntas de respuesta múltiple. Es uno de los instrumentos más utilizados para la evaluación de la depresión. Está compuesto por ítems relacionados con síntomas depresivos, como la desesperanza e irritabilidad, cogniciones como culpa, así como síntomas físicos relacionados con la depresión (fatiga, pérdida de peso y apetito sexual). El marco temporal hace referencia al momento actual y a la semana previa. Su contenido enfatiza más en el componente cognitivo de la depresión. Como otros instrumentos de evaluación de síntomas, su objetivo es cuantificar la sintomatología, no proporcionar un diagnóstico. En pacientes con enfermedades físicas la validez predictiva de la escala está peor establecida, ya que los ítems somáticos tienden a aumentar el número de falsos positivos.

La Escala para la Valoración de la Depresión de Hamilton (Hamilton Rating Scale for Depression, HRSD) fue desarrollado por Hamilton en $1960^{(63)}$. Su adaptación española ha sido realizada por Cordero y Ramos-Brieva en $1986^{(64)}$. Se trata de una escala diseñada para ser utilizada en pacientes diagnosticados previamente de depresión, con el objetivo de evaluar cuantitativamente la gravedad de los síntomas y valorar posibles cambios en la evolución del paciente. Su versión original constaba de 21 ítems, pero posteriormente se realizó una versión reducida con 17 ítems. Para la evaluación de la respuesta al tratamiento se ha definido como respuesta una disminución mayor o igual del $50 \%$ de la puntuación inicial de la escala, respuesta parcial como una disminución entre el 25-
$49 \%$ y una no respuesta como una reducción de menos del 25\%. La remisión se ha considerado con una puntuación menor o igual a 7. Su tiempo de aplicación oscila entre 15 y 20 minutos.

La Escala Autoaplicada de Depresión de Zung (Self-Rating Depression Scale, SDS) fue desarrollada por Zung et al. en $1965^{(65)}$ y adaptada a la población española por Conde et al. en $1970^{(66)}$. Al igual que la Escala para la Valoración de la Depresión de Hamilton da mayor peso al componente somático-conductual del trastorno depresivo.

\section{CALIDAD DE VIDA}

El tratamiento quirúrgico puede producir un descenso sustancial en el funcionamiento físico y un incremento en los síntomas físicos que pueden llevar a un descenso en la calidad de vida de los pacientes con diagnóstico de cáncer de pulmón no microcítico. En este sentido, Win et al. ${ }^{(67)}$ encuentran que la calidad de vida de los pacientes con tratamiento quirúrgico se ve disminuida significativamente al mes tras la intervención volviendo a niveles preoperatorios a los seis meses. Otros autores como Balduyck et al. ${ }^{(68)}$ indican que tras una lobectomía las puntuaciones en la calidad de vida vuelven a la línea base entre los tres y los seis meses. Por su parte, Paull et al. ${ }^{(69)}$ encuentran en sus investigaciones una disminución significativa de la calidad de vida de los pacientes en los tres primeros meses tras la intervención quirúrgica, que vuelve a los niveles previos en los nueve meses posteriores. Por el contrario, Kenny et al. ${ }^{(70)}$ concluyen en su estudio que la calidad de vida mejora en los dos años posteriores a la operación en aqueIlos pacientes que no presentan una recidiva, aunque prácticamente la mitad de los pacientes siguen experimentando síntomas y limitaciones funcionales. En vista de los resultados obtenidos por las diferentes in- 
vestigaciones descritas resulta de especial interés llevar a cabo estudios longitudinales que permitan conocer con exactitud el impacto del tratamiento quirúrgico en la calidad de vida de los pacientes y, el tiempo necesario para su recuperación.

Otros autores mencionan la existencia de factores predictores de una peor calidad de vida post-quirúrgica. RodríguezQuintana et al. ${ }^{(71)}$ describen en su estudio que la disnea es el síntoma principal asociado a déficit en la calidad de vida seguido de la fatiga y del dolor. Etxeberria et al. ${ }^{(72)}$ indican en sus resultados que los pacientes con un estadio más avanzado de la enfermedad muestran una peor calidad de vida en comparación con los pacientes con estadios más iniciales. Sarna et al. ${ }^{(73)}$ concluyen que el estado de ánimo deprimido, la comorbilidad y la disnea fueron factores que se asociaron con una peor calidad de vida física y emocional.

La evaluación de la calidad de vida representa un aspecto fundamental para evaluar los resultados de los tratamientos a corto y largo plazo, y constituye un factor pronóstico de supervivencia. Dentro de los instrumentos de evaluación específicos para el cáncer de pulmón entre los más utilizados en la investigación en este área se encuentra el cuestionario genérico EORTC QLQ C-30 desarrollado por Aaronson et al. en $1993^{(74)}$ y adaptado a la población española por Arrarás et al. en $1993^{(75)}$, y su módulo específico EORTC QLQ-LC13. El cuestionario genérico recoge los aspectos valorativos de diferentes áreas de la calidad de vida informados por el paciente. Incluye treinta ítems que se reparten en cinco escalas funcionales (funcionamiento físico, rol, emocional, social y cognitivo), tres escalas de síntomas (fatiga, dolor y nauseas o vómitos), una escala global de salud/calidad de vida y un número de ítems individuales que evalúan problemas económicos y síntomas de disnea, pérdida de apetito, insomnio, estreñimiento y diarrea. Las puntuaciones obtenidas por los pacientes se interpretan de manera que a una mayor puntuación en las escalas funcionales y en la global, le corresponde una mejor calidad de vida, y cuanto mayor sea la puntuación en la escala de síntomas y en los síntomas específicos, peor será la calidad de vida. La duración estimada de aplicación es de 15 minutos. El cuestionario EORTC QLQLC13 fue diseñado por Bergman et al. en $1994^{(76)}$ y adaptado a la población española por Arrarás et al. en $2000^{(77)}$ fue el primer módulo específico desarrollado. Se creó para valorar los síntomas específicos relacionados con el cáncer de pulmón y su tratamiento. El módulo consta de 13 preguntas. Se construyó de forma paralela al cuestionario general QLQ-C30. Incluye preguntas de evaluación relacionadas con el cáncer de pulmón y sus síntomas (tos, hemoptisis, disnea y dolor específico), relacionados con el tratamiento y sus efectos secundarios (dolor en la boca, disfagia, neuropatía periférica y alopecia) y analgésicos. Incorpora una escala multiítem para evaluar la disnea y una serie de ítems individuales que miden dolor, tos, sequedad de la boca, disfagia, neuropatía periférica, alopecia y hemoptisis. El sistema de puntuaciones es idéntico al de la escala general QLQ-C30.

El Instrumento de Evaluación Funcional de Calidad de Vida en Pacientes con Cáncer de Pulmón (Functional Assessment of Cancer Therapy-Lung Cancer, FACT-L) fue creado por Cella et al. en $1995^{(78)}$. Su traducción española se realizó tres años más tarde por de Cella et al. en $1998^{(79)}$. Consta de un cuestionario general (FACTG) desarrollado por Cella et al. en 1989(80), compuesto de 27 ítems generales. Su última versión está formada por 41 ítems de los cuales 34 hacen referencia a cinco dimensiones generales de la calidad de vida (física, social-familiar, emocional, funcional y relación con el médico), y siete ítems 
específicos de cáncer de pulmón (disnea, dificultad para respirar, tos, opresión en el pecho, apetito, pérdida de peso y función cognitiva).

El Cuestionario Lung Cancer Symptom Scale (LCSS) se desarrolló específicamente para el cáncer de pulmón. Fue desarrollada por Hollen et al. en $1993^{\left({ }^{(81)}\right.}$. Se centra principalmente en medir las dimensiones físicas y funcionales de dicha enfermedad. No valora los aspectos psicológicos y sociales, lo que puede limitar su utilidad. Presenta dos versiones, una para ser contestada por el paciente y otra para ser contestada por los profesionales sanitarios. Este aspecto permite realizar una comparación de ambas visiones de la enfermedad. La versión para el paciente mide los síntomas principales del cáncer de pulmón y además, el malestar físico, el nivel de actividad y la calidad de vida global. La versión para los profesionales sanitarios evalúa únicamente los síntomas. Carece de medida para parámetros sociales, espirituales y psicológicos.

A nivel general otros cuestionarios como Cuestionario de Salud SF-36 (The Short Form-36 Health Survey, SF-36) son utilizados igualmente para la evaluación de la calidad de vida en estos pacientes. El cuestionario de Salud SF-36 fue diseñado por Ware y Shebourne en $1992^{(82)}$ y adaptado a la población española por Alonso et al. en $1995^{(83)}$. Consta de 36 preguntas con las que pretender medir ocho conceptos genéricos de salud: función física, limitaciones de rol, dolor corporal, salud general, vitalidad, función social, rol emocional y salud mental. Detecta tanto estados positivos de salud como negativos, así como explora la salud física y la salud mental. Existe un elemento no incluido en estas ocho categorías, que explora los cambios experimentados en el estado de salud en el último año. Este ítem no se utiliza para el cálculo de ninguna de las escalas pero proporciona información útil sobre el cam- bio percibido. Existe una versión estandar que hace referencia al estado de salud en las cuatro semanas anteriores y una versión aguda que evalua la semana anterior. Se puede usar en conjunto con instrumentos específicos para médir resultados en la práctica clínica o de investigación.

El Índice de Karnofsky (Karnosky Performance Status, KPS) fue diseñado por Karnofsky et al. en $1948^{(84)}$. Se emplea para medir el nivel de actividad del paciente y las necesidades de atención médica. Se trata de una medida general de la autonomía del paciente y ha sido ampliamente utilizado como una evaluación general del paciente con cáncer. Es un elemento predictor independiente de mortalidad, tanto en patologías oncológicas como en no oncológicas. Sirve para la toma de decisiones clínicas y la valoración del impacto del tratamiento y la progresión de la enfermedad del paciente. La adaptación española fue realizada por Agra et al. en $1998^{(85)}$.

El Listado de Síntomas de Rotterdam (Rotterdam Symptom Checklist, RSCL) fue desarrollado por De Haes y Welvaart en $1985^{(86)}$ y adaptado a la población española por Agra y Badía en $1999^{(87)}$. Mide el impacto de los síntomas sobre la calidad de vida en pacientes con cáncer. Consta de 39 ítems que se agrupan en cuatro escalas: síntomas físicos (23 ítems), síntomas psíquicos (7), actividades de la vida diaria (8) y calidad de vida global (1).

\section{ESTRATEGIAS DE AFRONTAMIENTO}

En la actualidad el número de investigaciones sobre el uso de estrategias de afrontamiento en pacientes con cáncer de pulmón es limitado. Sin embargo, algunos autores han subrayado la importancia que la existencia o no de adecuadas estrategias de afrontamiento puede tener en el desarrollo de síntomas de ansiedad, depresión, dolor y en el descenso de la calidad de vida. En la investigación llevada a acabo 
por Rodríguez ${ }^{(88)}$ las estrategias de afrontamiento más utilizadas por los pacientes NSCLC fueron en primer lugar el fatalismo, seguido del espíritu de lucha y la negación. Los pacientes que utilizaban como estrategia de afrontamiento el espíritu de lucha presentaban menos preocupación ansiosa e indefensión. En este sentido, según el estudio de Uchitomi et al. ${ }^{(89)}$ el apoyo de los profesionales supone un impacto significativo en el espíritu de lucha de aquellos pacientes que no tienen historia de depresión previa.

Asimismo, Walker et al. ${ }^{(90)}$ muestran que la sintomatología depresiva se asociaba directamente al uso de la evitación como estrategia de afrontamiento y a un apoyo social que no respeta la autonomía del paciente.

Thornton et al. ${ }^{(91)}$ indican que aqueIlos pacientes que utilizan una estrategia de afrontamiento centrada en el problema eran más proclives a encontrar beneficios a la experiencia de la enfermedad.

Entre los instrumentos de evaluación específicos en este área destaca el Cuestionario de Ajuste Mental al Cáncer (Mental Adjustment to Cancer, MAC). Es la escala más empleada para evaluar las estrategias de afrontamiento en pacientes oncológicos. Fue desarrollada por Watson et al. en $1988^{(92)}$. Traducida y validada al español por Ferrero et al. en 1994(93). Consta de 40 ítems que contienen afirmaciones que intentan representar las diferentes maneras de interpretar y hacer frente a la enfermedad. Evalúa actitudes y reacciones cognitivas y conductuales. Existe una versión abreviada de 29 ítems, pero en la actualidad no se encuentra validada en nuestro país $^{(94)}$.

A nivel genérico, la Escala de Modos de Afrontamiento (Ways of Coping Inventory, WOC) fue desarrollada por Lazarus y Folkman en $1994^{(95)}$. Varios cuestionarios derivan del WOC, entre ellos el Inventario de Estrategias de Afrontamiento (CSI) y el Cuestionario de Afrontamiento al Estrés (COPE). Evalúa afrontamiento directo, apoyo, negociación, escape/evitación, planificación y solución de problemas, revaluación positiva, aceptación de responsabilidad y autocontrol. Su tiempo de aplicación es de 15 a 20 minutos. La traducción y validación a la población española fue realizada por Rodríguez et al. en $1992^{(96)}$.

El Cuestionario de Estilos de Afrontamiento del Estrés (COPE) es ampliamente utilizado. Fue desarrollado por Carver et al. en $1989^{(97)}$ y adaptado a la población española por Cruzado y Crespo en $1997^{(98)}$. Está basado en el modelo de estrés de Lazarus y en el modelo de autorregulación conductual de Carver y Séller. El cuestionario original consta de 60 ítems (4 ítems por cada una de las 15 escalas). Analiza 15 áreas o posibles estrategias: afrontamiento activo, planificación, búsqueda de apoyo social instrumental, búsqueda de apoyo social emocional, supresión de actividades distractoras, religión, reinterpretación positiva y crecimiento personal, refrenar el afrontamiento, aceptación, centrarse en las emociones y desahogarse, negación, desconexión mental, desconexión conductual, consumo de alcohol o drogas, humor. Carver en 1997(99) desarrollo su versión abreviada, el Brief COPE. Consta de 14 subescalas (afrontamiento activo, planificación, apoyo instrumental, uso de apoyo emocional, auto-distracción, desconexión conductual, reinterpretación positiva, negación, aceptación, religión, uso de sustancias, humor y auto-inculpación) con dos ítems cada una. Su tiempo de aplicación es de 15 a 20 minutos. El Inventario de Estrategias de Afrontamiento (Coping Strategies Indicator, CSI) fue desarrollado por Tobin et al. en $1989^{(100)}$ y adaptado en población general por Cano et al. en 2007 ${ }^{(101)}$. Consta de 40 ítems que miden el grado de utilización de estrategias de afrontamiento, diferenciando entre estrategias de afron- 
tamiento activo (resolución de problemas, restructuración cognitiva, expresión de emociones y apoyo social), y estrategias de afrontamiento pasivo (evitación de problemas, pensamientos ansiosos, autocrítica y retirada social). Sus respuestas son tipo Likert en cinco intervalos. Tiene una distribución jerárquica de las escalas. Ocho escalas primarias (resolución de problemas, restructuración cognitiva, apoyo social, expresión emocional, evitación de problemas, pensamiento desiderativo, retirada social y autocritica), cuatro secundarias dirigidas una a la acción centrada en el problema (resolución de problemas y restructuración cognitiva), otra a la acción centrada en la emoción (apoyo social y expresión de emociones), la tercera no dirigida a la acción centrada en el problema (evitación de problemas y pensamientos ansiosos) y la cuarta no dirigida a la acción centrada en la emoción (retirada social y autocrítica), y dos terciarias que surgen de la agrupación empírica de las secundarias (dirigida a la acción o activas y no dirigidas a la acción o pasivas). La escala consta de ítem adicional acerca de la autoeficacia percibida del afrontamiento.

El Cuestionario de Estrategias de Afrontamiento (Coping Strategies Questionnaire, CSQ), fue desarrollado a principios de la década de los 80 por Rosentiel y Keefe ${ }^{(102)}$. Su traducción española fue realizada por Rodríguez et al. en 2004 ${ }^{(103)}$. Está compuesto por 42 ítems agrupados en 7 escalas de 6 ítems cada una, más 2 ítems referidos al control que se ejerce sobre el dolor y la capacidad para disminuirlo. Cada ítem se puntúa de acuerdo con una escala de 7 puntos.

Debido a la escasez de estudios realizados en este grupo de pacientes y a los diferentes instrumentos de evaluación utilizados que no permiten la comparación entre las investigaciones no podemos concluir si las estrategias de afrontamiento son útiles en el screening de los pacientes con cáncer de pulmón. Es necesario llevar a cabo más estudios que nos permitan determinar la utilidad del screening en este área.

\section{CONCLUSIONES}

El cáncer de pulmón constituye un problema médico y también social, por su alta frecuencia y el elevado número de síntomas físicos y emocionales que presenta. La identificación de los niveles de malestar emocional en los pacientes con cáncer de pulmón sometidos a intervención quirúrgica es esencial para iniciar un tratamiento adecuado. Características sociodemográficas como el nivel educativo, el género o la edad, junto con características clínicas como el empeoramiento del estado funcional, la disnea o el consumo de tabaco, y de manera especialmente relevante el dolor, resultan factores de riesgo del malestar emocional. Igualmente, y a pesar de la falta de consenso ente las diferentes investigaciones descritas, los resultados obtenidos por los autores indican que el impacto del tratamiento quirúrgico supone un descenso sustancial en la calidad de vida de los pacientes. Finalmente, en cuanto a las estrategias de afrontamiento es necesario realizar más investigaciones para determinar su posible utilidad como elemento de screening.

Revisados estos aspectos resulta necesario establecer protocolos de screening con el objetivo de poder detectar aquellos pacientes con cáncer de pulmón no microcítico en riesgo de presentar malestar emocional durante el tratamiento. Para poder llevar a cabo este screening se deben tener en cuenta los factores de vulnerabilidad de cada persona. Igualmente la selección del instrumento es de gran importancia. Los instrumentos de medida específicos, con buenas propiedades psicométricas, cuyo tiempo de aplicación sea breve deben ser 
elegidos preferentemente. Esta medida de screening permitiría proporcionar al paciente la adecuada atención psicológica antes de que los problemas estén consolidados.

Las variables en las cuales deberíamos centrarnos son: el malestar emocional, la calidad de vida y el dolor. Los cuestionarios HADS, EORTC QLQ C30, LC13 y BPI pueden resultar de gran utilidad ya que constituye un protocolo de evaluación válido y sencillo de aplicar. Los momentos idóneos de evaluación son: el diagnóstico previo a la cirugía y después de ésta. Con los resultados obtenidos tras la medida de screening se debería derivar aquellos pacientes que presenten malestar emocional a la unidad de Psicooncología.

\section{REFERENCIAS BIBLIOGRÁFICAS}

1. Asociación Española de Afectados de Cáncer de Pulmón. Manifiesto mes del cáncer de pulmón [En línea] [Acceso el 1 de Septiembre de 2012]. Disponible en http: www.cancerpulmon.es

2. Tyczynski JE, Bray F, Parkin M. Lung cancer in Europe in 2000: Epidemiology, prevention and early detection. Lancet Oncol 2003;4:45-55. Doi:10.1016/S14702045(03)00960-4

3. Visbal AL, Williams BA, Nichols FC, Marks RS, Jett JR, Aubry MC, et al. Gender differences in non-small-cell lung cancer survival: An analysis of 4,418 patients diagnosed between 1997 and 2002. Ann Thorac Surg 2004;78:209-15. Doi:10.1016/j.athoracsur.2003.11.021

4. Hernández JR, Sánchez J. Epidemiología del cáncer de pulmón. En: Fernández Fau L, Freixinet Gilart J, editores. Tratado de cirugía torácica. Madrid: SEPAR Editores Médicos SA 2010;1559-79.

5. Parsons A, Daley A, Begh R, Aveyard $P$. Influence of smoking cessation after diagnosis of early stage lung cancer on prognosis: Systematic review of observational studies with meta-analysis. BMJ 2010;34:1-7. Doi:10.1136/bmj. b5569

6. Alberg AJ, Samet JM. Epidemiology of lung cancer. Chest 2003;123:21-49. Doi:10.1378/chest.123.1_suppl.21S

7. Robles AM, Shure D. Optimization of lung before pulmonary resection: Pulmonologist's perspective. Thorac Surg Clin 2004;14:295-304. Doi:10.1016/ S1547-4127(04)00018-0

8. López A, Callol L, Martín JL, Moreno N. Carcinoma broncogénico. En: Miguel J, Álvarez R, editores. Manual de Neumología Clínica. Madrid: ERGON 2009; 267-87.

9. Barreiro E. EPOC y cáncer de pulmón. Arch Bronconeumol 2008;44:399-401. Doi:10.1016/S0300-2896(08)72101-X

10. Piccirillo JF, Tierny RM, Costas I, Grove L, Spitznagel EL. Prognostic importance of comorbidity in a hospital-based cancer registry. JAMA 2004;291:2441-47.

11. Vasallo B. Historia de la estadificación del carcinoma broncogénico. En: Vasallo B, editores. Estadificación del carcinoma broncogénico. Madrid: Laboratorios Menari 2005.p.13-53

12. López A. Manifestaciones clínicas y diagnóstico del cáncer de pulmón. En: Fernández Fau L, Freixinet Gilart J, editores. Tratado de cirugía torácica. Madrid: SEPAR Editores Médicos 2010.p.1579-89

13. Scagliotti GV. Symptoms, signs and staging of lung cancer. Eur Respir Mon 2001;17:86-119.

14. Asociación Española Contra el Cáncer. El cáncer de pulmón. [En línea] [Acceso el 12 de Junio de 2012]. Disponible en: http://www.aecc.es

15. Castillo MC, Jorgelina G, Benavente EP, Mabel N. Neoplasia pulmonar. Revisión de datos clínicos, diagnósticos y terapéuticos. Rev Posgrado Vía Cátedra Med 2005;150:17-21.

16. Rami R, Saumench R, Serra M, Bidegain C. Estadificación del cáncer de pulmón. 
En: Fernández Fau L, Freixinet Gilart J, editores. Tratado de cirugía torácica. Madrid: SEPAR Editores Médicos 2010.p.1589-607

17. Paoletti L, Pastis NJ, Delinger $\mathrm{CH}$, Silvestri GA. A decade of advances in treatment of early-stage. Clin Chest Med 2011;32:82738. Doi:10.1016/j.ccm.2011.08.009

18. Fernández L, Wah Y, Moreno R, García JL. Cáncer de pulmón no de células pequeñas. En: Fernández Fau L, Freixinet Gilart J, editores. Tratado de cirugía torácica. Madrid: SEPAR Editores Médicos 2010;1625-67.

19. Arraigada R, Goldstraw P, Lechevalier I. Management of non-small cell lung cancer. En: Souhami RL, Tannock I, Hohenberger P, Horiot JC, editores. Oxford: Oxford University Press, 2002.

20. Putman JB. Pulmón (incluyendo embolia pulmonar y síndrome del desfiladero torácico). En: Townsend CM, Beauchamp RD, Mark B, Mattox KL, editores. Tratado de cirugía: Fundamentos biológicos de la práctica moderna. Madrid: Elsevier España 2005.p.1763-812

21. Bootom R, Jones $M$, Thatcher N. Management of lung cancer in elderly patients. Thorax 2003;58:711-20. Doi:10.1136/tho$\operatorname{rax} .58 .8 .711$

22. Pagano E, Filippini C, Di Cuonzo D, Ruffini E, Zanetti R, Rosso S, et al. Factors affecting pattern of care and survival in a population-based cohort of non-smallcell lung cancer incident cases. Cancer Epidemiol 2010;34:483-9. Doi:10.1016/j. canep.2010.04.002

23. Eguino A, Fernández B, García G, García JA. Cáncer de pulmón: Una guía práctica. Asociación Española Contra el Cáncer, 2005.

24. Rogers ML, Duffy JP. Surgical aspects of chronic post-thoracotomy pain. Eur J Cardiothorac Surg 2000;18:711-6. Doi:10.1016/S1010-7940(00)00569-8

25. Sugimura $H$, Yang $P$. Long-term survivors in lung cancer: A review. Chest 2006;129:1088-97. Doi:10.1378/ chest.129.4.1088
26. Wildgaard K, Ravn J, Kehlet H. Chronic post-thoracotomy pain: A critical review of pathogenic mechanisms and strategies for prevention. Eur J Cardiothorac Surg 2009;36: 170-80. Doi:10.1016/j. ejcts.2009.02.005

27. Karmakar SA, Ho MH. Postthoracotomy pain syndrome. Thorac Surg Clin 2004;14: 345-52. Doi:10.1016/S15474127(04)00022-2

28. Macrae WA. Chronic post-surgical pain: 10 years on. Br J Anaesth 2008;101:77-86. Doi: 10.1093/bja/aen099

29. Hazelrigg SR, Cetindag IB, Fullerton J. Acute and chronic pain syndromes after thoracic surgery. Surg Clin North Am 2002;82:849-65. Doi:10.1016/S00396109(02)00031-2

30. Cleeland CS. Pain assessment in cancer. Effect of cancer on quality of life. Boca Raton: CRC, 1991.

31. Badía XL, Muriel C, Gracia CS, Muriel C, Gracia A, Perulero N, et al. Validación española del cuestionario Brief Pain Inventory en pacientes con dolor de causa neoplásica. Med Clin 2003;120:52-9.

32. Zabora J, Brintzenhofeszoc K, Curbow B, Hooker C, Piantadosi S. The prevalence of psychological distress by cancer site. Psychooncology 2001;10:19-28. Doi:10.1002/1099$1611(200101 / 02) 10: 1<19:$ :AID PON501>3.0.CO;2-6

33. Camacho S. El cáncer de pulmón. En DieTrill M, editores. Psico-Oncología. Madrid: Ades Ediciones 2003;207-24.

34. Uchitomi Y, Mikami I, Nagai K, Nishiwaki Y, Akechi T, Okamura H. Depression and psychological distress in patients during the year after curative resection of non-smallcell lung cancer. J Clin Oncol 2003;21:6977. Doi:10.1200/JCO.2003.12.139

35. Sarna L, Cooley ME, Brown JK, Williams RD, Cjernecky C, Padilla G, et al. Quality of life and health status of dyads of women with lung cancer and family members. Oncol Nurs Forum 2006;33:1109-16. 
Doi:10.1188/06.ONF.1109-1116

36. Graves KD, Arnold SM, Love CL, Kirsh $\mathrm{KL}$, Moore PG, Passik SD. Distress screening in a multidisciplinary lung cancer clinic: Prevalence and predictors of clinically-significant distress. Lung Cancer 2007;55:215-24. Doi:10.1016/j.lungcan.2006.10.001

37. Hopwood P, Stephens RJ. Depression in patients with lung cancer: Prevalence and risk factors derived from quality of life data. J Clin Oncol 2000;18:893-903.

38. Uchitomi Y, Mikami I, Ngai K, Nishiwaki Y, Akechi T, Okamura H. Depression and psychological distress in patients during the year after curative resection of non-smallcell lung cancer. J Clin Oncol 2003;21:6977. Doi:10.1200/JCO.2003.12.139

39. Smith EL, Hann DM, Ahles TA, Furstenbrg CT, Mitchell TA, Meyer L, et al. Dyspnea, anxiety, body consciousness and quality of life in patients with lung cancer. J Pain Symptom Manage 2001;21:323-29. Doi:10.1016/S0885-3924(01)00255-X

40. Handy JR, Asaph JW, Stokan L, Redd CE, Koh S, Brooks G, et al. What happens to patients undergoing lung cancer surgery? Outcomes and quality of life before and after surgery. Chest 2002;122:21-30. Doi:10.1378/chest.122.1.21

41. Kurzt ME, Kurzt JC, Stommel M, Given CW, Give B. Predictors of depressive symptomatology of geriatric patients with lung cancer: A longitudinal analysis. Psychooncology 2002;11:12-22. Doi:10.1007/s00520-001-0338-8

42. Henoch I, Bergman B, Gustafsson M, Gaston-Johansson F, Danielson E. The impact of symptoms, coping capacity and social support on quality of life experience over time in patients with lung cancer. J Pain Symptom Manage 2007;34:370-79. Doi:10.1016/j.jpainsymman.2006.12.005

43. Buchanan D, Milroy R, Baker L, Thompson AM, Levack PA. Perceptions of anxiety in lung cancer patients and their support network. Support Care Cancer 2010;18:29-
36. Doi:10.1007/s00520-009-0626-2

44. LoConte NK, Else-Quest NM, Eickhoff J, Hyde J, Schiller JH. Assessment of guilty and shame in patients with non-smallcell lung cancer compared with patients with breast and prostate cancer. Clin Lung Cancer 2008;9:171-8. Doi:10.3816/ CLC.2008.n.026

45. Rouhani M, Holland JC. Aspectos psiquiátricos del cáncer. En: Gelder MG, López-lbor JJ, Andersen N, editores. Tratado de Psiquiatría. Madrid: Ars Médica 2003;1407-15.

46. Angelino AF, Treisman GJ. Major depression and desmoralization in cancer patients: Diagnostic and treatment considerations. Support Care Cancer 2001;9:344-9. Doi:10.1007/s005200000195

47. Zigmond AS, Snaith RP. The hospital anxiety and depression scale. Acta Psychiatr Scand 1983;67:361-70. Doi:10.1111/j.1600-0447.1983.tb09716.x

48. Tejero A, Guimerá EM, Farré JM, Peri JM. Uso clínico del HAD (Hospital Anxiety and Depression Scale) en población psiquiátrica: Un estudio de su sensibilidad, fiabilidad y validez. Rev Depto Psiquiatría Facultad de Med Barna 1986;13:233-8.

49. Spielberger, CD. Manual for the State-Trait Anxiety Inventory (STAI). PaloAlto, CA: Consulting Psychologist Press, 1983.

50. Spielberger CD, Gorsuch RL, Lushene R. Manual del Cuestionario de Ansiedad Estado/Rasgo (STAI). Madrid: TEA Ediciones, 1982.

51. Beck AT, Epstein N, Brown G, Steer RA. An inventory of measuring clinical anxiety: Psychometric properties. J Consult Clin Psych 1988;56:893-7. Doi:10.1037//0022006X.56.6.893

52. Sanz J, Navarro ME. Propiedades psicométricas de una versión española del Inventario de Ansiedad de Beck (BAI) en estudiantes universitarios. Ansiedad Estrés, 2003;9:59-84.

53. MCNair DM, Lorr M, Droppleman LF. Manual for the Profile of Mood States. 
San Diego, CA: Educational and Industrial Testing Services, 1971.

54. Arce C, Andrade EM, Seoane P. Adaptación al español del cuestionario perfil de los estados de ánimo en un muestra de deportistas. Psicothema 2002;14:708-13.

55. Radloff LS. The CES-D Scale: A self-report depression scale for research in the general population. Appl Psych Meas 1977;1:385401. Doi:10.1177/014662167700100306

56. Chorot P, Sandín B. Escala de depresión CESDS. Madrid: UNED, 1991.

57. Derogatis LR, Melisaratos N. The Brief Symptom Inventory: An introduction report. Psychol Med 1983;13:595-605. Doi:10.1017/S0033291700048017

58. Aragón N, Bragado MC, Carrasco I. Fiabilidad y estructura factorial del Inventario Breve de Síntomas (BSI) en adultos. Psicol Conduct 2000;8:77-83.

59. Derogatis LR. SCL-90R: Administration, scoring and procedures. Manual-I for the Revised Version. Baltimore, Johns Hopkins, 1977

60. Beck AT, Ward $\mathrm{CH}$, Mendelson $\mathrm{M}$, Mock J, Erbaugh J. An inventory of measuring depression. Arch Gen Psychiat 1961;4:53-63. Doi:10.1001/ archpsyc.1961.01710120031004

61. Vázquez C, Sanz J. Fiabilidad y validez de la versión española del Inventario para la Depresión de Beck de 1978 en pacientes con trastornos psicológicos. Clin Salud 1999;10:59-68.

62. Beck AT, Steer RA, Ball R, Ranieri W. Comparison of Beck Depression Inventories IA and II in psychiatric outpatients. J Pers Assess 1996;67: 588-97. Doi10.1207/ s15327752jpa6703_13.

63. Hamilton M. A rating scale for depression. J Neurol Neurosurg Psychiatry 1960;23:56. Doi:10.1136/jnnp.23.1.56

64. Cordero A, Ramos-Brieva JA. A new validation of the Hamilton Rating Scale for Depression. J Psychiat Res 1988;22:21-8. Doi:10.1016/0022-3956(88)90024-6

65. Zung WK, Richards CB, Short MJ. A Self-
Rating Depression Scale. Arch Gen Psychiatry 1965;13:508-15. Doi:10.1001/ archpsyc.1965.01720310065008

66. CondeV, Escribá JA, Izquierdo J. Evaluación estadística y adaptación castellana de la escala autoaplicada para la depresión de Zung. Arch Neurobiol 1970;33:185-206.

67. Win T, Wells FC, Ritchie AJ, Munday $H$, Laroche CM, Sharples L. Effect of lung cancer surgery on quality of life. Thorax 2005;60:234-8. Doi:10.1136/ thx.2004.031872

68. Paull DE, Thomas ML, Meade GE, Updyke GM, Arocho MA, Chin HW, et al. Determinants of quality of life in patients following pulmonary resection for lung cancer. Am J Surg 2006;192:565-71. Doi:10.1016/j. amjsurg.2006.08.019

69. Kenny PM, King MT, Viney RC, Boyer MJ, Pollicino CA, McLean JM, et al. Quality of life and survival in the 2 years after surgery for non-small-cell lung cancer. J Clin Oncol 2008;26:233-41. Doi:10.1200/ JCO.2006.07.7230

70. Balduyck B, Hendriks J, Lauwers P, Nia PS, Schil PV. Quality of life evolution after lung cancer surgery in septuagenarians: A prospective study. Eur J CardioThorac 2009;35:1070-5. Doi:10.1016/j. ejcts.2009.01.050

71. Rodríguez-Quintana R, Hernando-Trancho F, Cruzado JA, Olivares ME, Jamal I, Laserna P. Evaluación de la calidad de vida, estado emocional y estrategias de afrontamiento en pacientes con enfermedad neoplásica pulmonar. Psicooncología 2012;9:95-112. Doi: 10.5209/rev_PSIC.2012.v9.n1.39140

72. Etxeberria Y, Pérez-Izquierdo J, Badiola C, Quintana JM, Padierna A, Aróstegui I, et al. Evaluación de la calidad de vida en pacientes con cáncer de pulmón. Gac Sanit 2001;15:25-31.

73. Sarna L, Cooley ME, Brown JK, Chernecky C, Padilla G, Danao L et al. Women with lung cancer: Quality of life after thoracotomy. Cancer Nurs 2010;33:85-92. Doi:10.1097/NCC.0b013e3181be5e51 
74. Aaronson NK, Ahmedzai S, Bergman B, Bullinger M, Cull A, Duez NJ et al. The European Organization for Research and Treatment of Cancer QLQ-C30: A quality of life instrument for use in international clinical trials in oncology. J Natl Cancer Inst 1993;85:365-76. Doi:10.1093/ jnci/85.5.365

75. Arrarás LI, Illaramendi JJ, Valerdi JJ. El cuestionario de calidad de vida de la EORTC. Estudio estadístico de validación con una muestra española. Rev Psicol Salud 1995;7:13-33.

76. Bergman B, Aaronson NK, Ahmedzai S, Kaasa S, Sullivan M. The EORTC QLQLC13: A modular supplement to the EORTC core quality of life questionnaire (QLQ-C30) for use in lung cancer clinical trials. Eur J Cancer 1994;30:635-42. Doi:10.1016/0959-8049(94)90535-5

77. Arrarás JI, Pruja E, Marcos M, Tejedor M, Illarramndi JJ, Vera R, et al. El cuestionario de Calidad de Vida para cáncer de pulmón de la EORTC QLQ-LC13. Estudio de validación para nuestro país. Oncología 2000;23:127-34.

78. Cella DF, Bonomi AE, Lloyd SR, Tulsky DS, Kaplan E, Bonomi P. Reliability and validity of the functional assessment of cancer therapy-lung (FACTL) quality of life instrument. Lung Cancer 1995;12:199220. Doi:10.1016/0169-5002(95)00450-F

79. Cella D, Hernández L, Bonomi AE, Corona $M$, Vaquero $M$, Shiomoto $G$, et al. Spanish language translation and initial validation of the functional assessment of cancer therapy quality of life instrument. Med Care 1998;36:1407-18. Doi: 10.1097/00005650-199809000-00012

80. Cella DF, Tross S, Orav JE. Mood states of patients after the diagnosis of cancer. J Psychosoc Oncol 1989;7:45-54. Doi:10.1300/J077v07n01_04

81. Hollen PJ, Gralla RJ, Kris MG, Potanovich LM. Quality of life assessment in individuals with lung cancer: Testing the Lung Cancer Symptom Scale (LCSS). Eur
J Cancer 1993;29:S51-8. Doi:10.1016/ S0959-8049(05)80262-X

82. Ware JE, Sherbourne CD. The MOS 36 item short-form health survey (SF-36). Conceptual framework and items selection. Med Care 1992;30:473-83. Doi:10.1097/00005650-19920600000002

83. Alonso J, Prieto L, AntóJM. La versión española del SF-36 Health Survey (Cuestionario de salud SF-36): Un instrumento para la medida de los resultados clínicos. Med Clin 1995;104:771-6.

84. Karnofsky D, Abelmann W, Craver L, Burchenal J. The use of nitrogen mustard in the palliative treatment of cancer. Cancer 1948;1:634-56. Doi:10.1002/1097$0142(194811) 1: 4<634$ : : A I D CNCR2820010410>3.0.CO;2-L

85. Agra $Y$, Badía $X$, Gil A. Instrumentos para la medición de la calidad de vida en los pacientes con cáncer. Med Clin 1998;110:703-8.

86. De Haes JC, van Knippenberg FC, Neijt JP. Measuring psychological and physical distress in cancer patients: Structure and application of the Rotterdam Symptom Checklist. Brit J Cancer 1990;62:1034-8. Doi:10.1038/bjc.1990.434

87. Agra Y, Badía X. Evaluación de las propiedades psicométricas de la versión española del Rotterdam Symptom Checklist para medir calidad de vida en personas con cáncer. Revista Española de Salud Pública 1999;73:35-44. Doi:10.1590/S113557271999000100005

88. Rodríguez-Quintana R. Evaluación de la calidad de vida, estado emocional y estrategias de afrontamiento en pacientes con enfermedad neoplásica pulmonar. Diploma de Estudios Avanzados. Facultad de Psicología, Universidad Complutense, Madrid, España, 2010.

89. Uchitomi Y, Mikami I, Kugaya A, Nakano T, OkuyamaT, AkechiT, etal. Physician support and patient psychological responses after surgery for non-small cell lung carcinoma: 
A prospective observational study. Cancer 2001;92:1926-35. Doi:10.1002/1097$0142(20011001) 92: 7<1926:$ :AID CNCR1711>3.0.CO;2-L

90. Walker MS, Zona DS, Fisher EB. Depressive symptoms after lung cancer surgery: Their relation to coping style and social support. Psychooncology 2006;15:68493. Doi:10.1002/pon.997

91. Thornton AA, Owen JE, Kernstine K, Kocywas M, Grannis F, Cristea M, et al. Predictors of finding benefit after lung cancer diagnosis. Psychooncology 2012;4:365-73. Doi: 10.1002/pon.1904.

92. Watson $\mathrm{M}$, Greer S, Young J, Inayat Q, Burgess C, Robertson B. Development of a questionnaire measure of adjustment to cancer: The MAC scale. Psychol Med 1988;18: 203-9. Doi:10.1017/ S0033291700002026

93. Ferrero J, Barreto M, Toledo M. Mental adjustment to cancer and quality of life in breast cancer patients: An exploratory study. Psychooncology 1994;3:223-32. Doi:10.1002/pon.2960030309

94. Watson M, Law M, Dos Santos M, Greer S, Brauch S, Bliss J. The Mini-Mac: futher development of the Mental Adjustment to Cancer Scale. Journal of Psychosocial Oncology 1994;12:33-46. Doi: 10.1300/ J077V12N03.03

95. Folkman S, Lazarus RS. Manual for the Ways of Coping Questionnaire. Palo Alto. CA: Consulting Psychologist Press, 1998.

96. Rodríguez-Marín J, Terol MC, López-Roig S, Pastor MA. Evaluación del afrontamiento del estrés: Propiedades psicométricas del cuestionario de formas de afrontamiento de acontecimientos estresantes. Rev Psicol Salud 1992;4:59-84.

97. Carver CS, Scheier MF, Weintraub JK. Assessing coping strategies: A theoretically based approach. J Pers Soc Psychol 1989;56:267-83. Doi:10.1037//00223514.56.2.267

98. Crespo M, Cruzado JA. La evaluación del afrontamiento: Adaptación española del cuestionario COPE con una muestra de estudiantes universitarios. Anal Modif Cond 1997;23:797-830.

99. Carver CS. You want to measure coping but your protocol's too long: Consider the Brief COPE. Int J Behav Med 1997;4:92100. Doi:10.1207/s15327558ijbm0401_6

100. Tobin DL, Holroyd KA, Reynolds RV, Kigal JK. The hierarchical factor structure of the Coping Strategies Inventory. Cognitive Ther Res 1989;13:343-61. Doi:10.1007/ BF01173478

101. Rodríguez L, Cano FJ, Blanco A. Evaluación de las estrategias de afrontamiento del dolor crónico. Actas Esp Psiquiatr 2004;32:82-91.

102. Rosentiel AK, Keefe FJ. The use of cognitive coping strategies in chronic low back pain patients: Relationship to patient characteristics and current adjustment. Pain 1983;17:33-44. Doi: 10.1016/03043959(83)90125-2

103. Cano FJ, Rodríguez L, Garcia J. Spanish version of the Coping Strategies Inventory. Actas Esp Psiquiatr 2007;35:29-39. 